\section{Estado nutricional e fatores associados em idosos do Município de Viçosa, Minas Gerais, Brasil}

\author{
Nutritional status and associated factors among \\ the elderly in Viçosa, Minas Gerais State, Brazil
}

\section{Abstract}

This cross-sectional study in 2009 focused on the prevalence of underweight and overweight and associated factors in a sample of 621 elderly individuals in Viçosa, Minas Gerais State, Brazil. Data were obtained through home interviews on nutritional status and socio-demographic and health conditions. Of the total sample, $53.3 \%$ were females. Median BMI was $26.40 \mathrm{~kg} /$ $m^{2}$ (range $=15.20-46.82$ ) and was lower for men than for women. Prevalence of overweight was high (45\%; 95\%CI: 40\%-49\%), decreased with age, was positively associated with female gender and history of arthritis/arthrosis, and was negatively associated with age 80 years or older and smoking. Prevalence of underweight was high in men (18.2\%), increased with age, and was positively associated with worse self-rated health. The findings highlight the importance of health strategies that favor lifestyle changes and healthy eating habits.

Nutritional Status; Body Mass Index; Obesity; Aged
Clarissa de Matos Nascimento 1

Andréia Queiroz Ribeiro ${ }^{1}$

Rosângela Minardi Mitre Cotta 1

Francisco de Assis Acurcio 2

Sérgio Viana Peixoto 2

Silvia Eloiza Priore 1

Sylvia do Carmo Castro Franceschini 1

\section{Introdução}

Em decorrência do processo de envelhecimento verifica-se uma mudança no perfil epidemiológico e nutricional da população com o predomínio de agravos de saúde específicos, tais como doenças crônicas não transmissíveis e incapacidades, resultando em maior demanda pelos serviços de saúde e maior consumo de medicamentos 1,2 .

O processo de envelhecimento é caracterizado por alterações metabólicas, fisiológicas, psicológicas e bioquímicas. Dentre essas alterações na composição corporal tem-se a redistribuição da gordura corporal, com acúmulo visceral, enquanto a massa muscular diminui nos membros inferiores e superiores 3 .

Estudos epidemiológicos sobre estado nutricional, em idosos, utilizam o índice de massa corporal para avaliar o baixo peso e o excesso de peso, que são importantes fatores de risco para a morbidade neste grupo etário 4.

Dados da Pesquisa Nacional sobre Saúde e Nutrição (PNSN-1989) e da Pesquisa sobre Padrões de Vida (PPV 1996-1997) indicaram prevalências maiores de excesso de peso em relação ao baixo peso entre os idosos brasileiros. Com relação ao sexo, a PNSN encontrou prevalência de sobrepeso menor entre os homens $(24,7 \%)$ do que entre as mulheres (32\%). Perfil semelhante foi observado pela PPV ( $31,9 \%$ vs. $32,7 \%$, respectivamente). Já o baixo peso foi maior no sexo masculino (7,8\% vs. 6\%), de acordo com a PPV 5,6. 
No Brasil, poucos estudos têm focalizado os determinantes das alterações nutricionais em idosos, apesar de sua importância para o planejamento de ações em saúde. Sabe-se que dentre as condições associadas às alterações do estado nutricional destacam-se a hipertensão arterial, diabetes mellitus 7 , dislipidemias 8 , artrite ${ }^{9}$, polifarmácia e maior número de internações 7 .

Nesse contexto, o presente trabalho objetivou determinar a prevalência e os fatores associados ao baixo peso e ao excesso de peso, em idosos do Município de Viçosa, Minas Gerais, Brasil.

\section{Materiais e métodos}

Estudo observacional, de corte transversal, conduzido no Município de Viçosa no período de junho a dezembro de 2009, com idosos de 60 anos e mais de idade, desenvolvido pelo Departamento de Nutrição e Saúde da Universidade Federal de Viçosa. Localizado na região da zona da mata mineira, o município contava, no ano de 2007, com uma população de 70.404 habitantes, dos quais 7.034 tinham 60 anos ou mais de idade.

\section{População alvo e amostra}

A população alvo deste estudo foi constituída pelos idosos, com 60 ou mais anos de idade, residentes no Município de Viçosa, incluindo as zonas urbana e rural. Essa população foi recenseada durante a Campanha Nacional de Vacinação do Idoso, no período de abril a maio de 2008. Com o objetivo de identificar os não participantes da campanha de vacinação e complementar a base cadastral, procedeu-se à confrontação e junção desse banco de dados com outros disponíveis no município, a saber: o dos Servidores da Universidade Federal de Viçosa, ativos e aposentados; os cadastros dos idosos do Programa Saúde da Família (PSF), do serviço de fisioterapia municipal, do centro de saúde da mulher, do serviço psicossocial, da unidade de atendimento, do HiperDia e da Policlínica. Após essa junção dos bancos, o registro de pessoas com 60 anos e mais totalizou 7.980, que serviu de base para a obtenção da amostra. Não fizeram parte do presente estudo os idosos institucionalizados.

O cálculo do tamanho amostral considerou um nível de 95\% de confiança, prevalências estimadas de $50 \%$ e variabilidade de $4 \%$. Assim, a amostra seria de 558 idosos, à qual se acrescentou $20 \%$ para cobrir possíveis perdas, totalizando 670 pessoas a serem estudadas. Ocorreram perdas de informação por recusa $(3,6 \%)$ e por motivos inevitáveis para a realização das entrevistas $(3,7 \%)$. Considerou-se perdas inevitáveis as situações em que foram sorteados indivíduos que haviam falecido (1,3\%), endereços não localizados $(1,2 \%)$ e aqueles que se mudaram para residências de difícil localização e para outros municípios $(1,2 \%)$. Assim, foram efetivamente estudados 621 idosos.

\section{Coleta de dados}

As entrevistas foram domiciliares e, preferencialmente, pré-agendadas. Duplas de entrevistadores, previamente treinados, localizavam os domicílios e visitavam os idosos com o objetivo de explicar os objetivos da pesquisa. Esses eram então convidados a participar e agendava-se dia e horário para os que aceitavam.

As informações foram obtidas usando-se um questionário semiestruturado com a maioria das perguntas fechadas e pré-codificadas. O questionário era aplicado diretamente ao idoso, mas caso ele expressasse alguma dificuldade o respondente próximo o auxiliava. Cerca de 25,7\% dos idosos foram auxiliados em alguma seção do questionário, sendo a grande maioria dos auxílios prestada por familiares (95\%).

Medidas de peso e altura foram realizadas para avaliação antropométrica. O peso foi aferido em balança portátil (eletrônica digital - LC 200pp, Marte Balanças e Aparelhos de Precisão Ltda., Santa Rita do Sapucaí, Brasil), com capacidade de $199,95 \mathrm{~kg}$ e precisão de $50 \mathrm{~g}$, com os idosos vestindo roupas leves, sem sapatos e sem agasalhos. Eles ficaram em posição ortostática, com os braços estendidos e o olhar voltado para o horizonte 10 .

A estatura foi aferida com o auxílio de um estadiômetro portátil com extensão de 2,13 metros, dividido em centímetros e subdividido em milímetros (Alturaexata, Belo Horizonte, Brasil). Para aferição da estatura os idosos estavam descalços, com os calcanhares juntos, em posição ereta, encostados no estadiômetro e com o olhar fixo na altura da linha do horizonte 10 .

As medidas de peso e altura que apresentaram problemas posturais, amputação da perna ou dificuldade de manter-se de pé foram excluídas, as quais corresponderam a 11,3\% (70) e 2,6\% (16), respectivamente, mantendo-se a análise das outras informações referentes a esses idosos.

\section{Variáveis do estudo}

A variável dependente analisada foi o índice de massa corporal (IMC) calculado com base na divisão do peso corporal em quilogramas pela estatura em metro elevada ao quadrado $\left(\mathrm{kg} / \mathrm{m}^{2}\right)$. Os pontos de corte adotados para avaliar o estado nutricional foram os propostos por Lipschitz 11: 
baixo peso $\left(<22 \mathrm{~kg} / \mathrm{m}^{2}\right)$, eutrofia $\left(22-27 \mathrm{~kg} / \mathrm{m}^{2}\right)$ e sobrepeso ( $\left.>27 \mathrm{~kg} / \mathrm{m}^{2}\right)$.

As variáveis independentes analisadas foram:

a) Características sociodemográficas: idade (6069 anos, 70-79 e 80 anos e mais), sexo (masculino, feminino), escolaridade (nunca estudou, até as séries iniciais do Ensino Fundamental, e séries finais do Ensino Fundamental ou mais), e renda (menor ou igual à mediana e maior que a mediana);

b) Indicadores das condições de saúde: percepção da própria saúde (muito boa/boa, regular, ruim/muito ruim), morbidade referida (história de diabetes, pressão alta, dislipidemias e artrite/ artrose), número de morbidades autorreferidas (até quatro doenças, e cinco ou mais doenças autorrelatadas), número de medicamentos consumidos (até quatro, e cinco ou mais), hábitos de vida: prática de atividade física (sim ou não), tabagismo (sem história de tabagismo, ex-tabagista, tabagista atual);

c) Indicadores de uso de serviços de saúde: número de internações hospitalares 12 meses antes da realização da entrevista (nem uma vez, uma vez ou mais). As morbidades foram investigadas baseando-se na pergunta sobre se alguma vez na vida um médico ou outro profissional de saúde relatou alguma condição em particular, totalizando 12 morbidades de interesse. Com base nessa questão utilizou-se a variável número de morbidades autorreferidas, sendo categorizada em até quatro, e cinco ou mais. Além disso, dentre as 12 morbidades definidas quatro foram consideradas de interesse: diabetes, hipertensão, dislipidemias e artrite/artrose.

\section{Análise dos dados}

A análise descritiva das variáveis foi apresentada por meio de média e desvio-padrão e/ou mediana seguida pelos valores mínimo e máximo. A normalidade da distribuição das variáveis foi avaliada usando-se o teste de Kolmogorov-Smirnov 12 . Foram estimadas as prevalências de baixo peso e excesso de peso. As análises foram estratificadas segundo o sexo (masculino e feminino) e a faixa etária (60-69 anos, 70-79 anos, $\geq 80$ anos).

O teste de Mann Whitney foi realizado para comparação do IMC entre os sexos. Realizou-se o teste de Kruskal Wallis complementado com o procedimento de comparações múltiplas de Dunn's para comparação dos valores de IMC entre as faixas etárias dentro de cada sexo. O teste do qui-quadrado de tendência linear foi utilizado para comparar prevalências de baixo peso e sobrepeso de acordo com o a faixa etária dentro de cada sexo ${ }^{12}$. A análise de regressão logística mul- tinomial foi empregada para obter estimativas da odds ratio e os respectivos intervalos de $95 \%$ de confiança. Neste estudo, a comparação entre as categorias foi feita entre os que apresentaram baixo peso e excesso de peso em comparação aos eutróficos. As variáveis que na análise bivariada multinomial se associaram à variável dependente com nível de significância menor que 0,20 foram incluídas no modelo logístico multivariado. O modelo final foi obtido utilizando-se o método de "trás para frente", com teste de significância da eliminação da variável em cada estágio. As variáveis que se associaram à variável dependente com nível de significância menor que 0,05 permaneceram no modelo final.

Para o armazenamento dos dados foi utilizado o software Epi Info versão 6.04 (Centers for Disease Control and Prevention, Atlanta, Estados Unidos), e para análise de dados foram usados os softwares Stata versão 7.0 (Stata Corp., College Station, Estados Unidos) e SigmaStata versão 2.03 (Jandel Scientific, Califórnia, Estados Unidos). Para rejeição da hipótese de nulidade adotou-se como nível de significância estatística o valor $\mathrm{p}<$ 0,05 para todas as comparações.

O estudo foi aprovado pelo Comitê de Ética em Pesquisa com Seres Humanos da Universidade Federal de Viçosa.

\section{Resultados}

Dos 621 idosos entrevistados, 53,3\% $(n=331)$ eram do sexo feminino. A mediana de idade foi de 69 anos, variando entre 60 e 98. A Tabela 1 mostra a distribuição de IMC para homens e mulheres, de acordo com a faixa etária. A mediana total do IMC foi de 26,40 (mínima 15,20 e máxima 46,82), sendo menor para os homens $(\mathrm{p}<0,001)$. A redução do IMC de acordo com a idade foi observada nos homens $(\mathrm{p}<0,042)$.

A prevalência de excesso de peso foi de $45 \%$ (IC95\%: 40,8\%-49,1\%) e a de baixo peso de $13,6 \%$ (IC95\%: 10,7\%-16,8\%). A prevalência de baixo peso foi significantemente maior entre os homens $(18,2 \%)$ quando comparada à das mulheres $(9,0 \%)$. Observa-se a tendência significante de aumento da prevalência de baixo peso somente entre os homens ( $\chi^{2}$ de tendência $=5,58$; $\mathrm{p}=$ 0,018) (Figura 1).

A prevalência de excesso de peso foi maior entre as mulheres $(54,5 \%)$ quando comparada à dos homens (29,6\%), e diminuiu com a idade em ambos os sexos (Figura 2). No entanto, redução significante foi observada somente dentre as mulheres ( $\chi^{2}$ de tendência $=4,66 ; p=0,030$ ).

$\mathrm{Na}$ Tabela 2, encontram-se os resultados da análise bivariada do baixo peso e excesso de peso 
Índice de massa corporal (IMC) de acordo com o sexo e o grupo de idade dos idosos. Viçosa, Minas Gerais, Brasil, 2009.

\begin{tabular}{lccc}
\hline Sexo e faixa etária & $\mathbf{n} *$ & Média \pm DP & Me (min-máx) \\
\hline $\begin{array}{l}\text { Homens (anos) } \\
\text { 60-69 a }\end{array}$ & 148 & & \\
70-79 b & 99 & $26,4 \pm 4,7$ & $25,9(15,7-41,6)$ \\
80 e mais c & 27 & $25,3 \pm 4,0$ & $25,2(16,8-41,7)$ \\
Total ** & 274 & $23,8 \pm 3,9$ & $23,0(15,9-29,8)$ \\
Mulheres (anos) & & $25,7 \pm 4,4$ & $25,4(15,7-41,7)$ \\
60-69 d & 156 & & \\
70-79 e & 89 & $28,7 \pm 5,5$ & $28,0(17,1-46,8)$ \\
80 e mais $f$ & 32 & $27,4 \pm 5,0$ & $27,3(15,2-43,5)$ \\
Total ** & 277 & $27,2 \pm 5,8$ & $26,0(18,5-46,1)$ \\
\hline
\end{tabular}

DP: desvio padrão; máx: valor máximo; Me: mediana; min: valor mínimo.

* O total geral $(n=551)$ é menor que o total da amostra em função de perdas nas medidas antropométricas;

** Mann-Whitney: $p<0,001$ entre os sexos.

Teste de Kruskal Wallis: homens $(p<0,042): a=b ; b=c ; a>c ;$ mulheres: $d=e=f$.

Figura 1

Prevalência de baixo peso entre os idosos, de acordo com o gênero e a faixa etária.

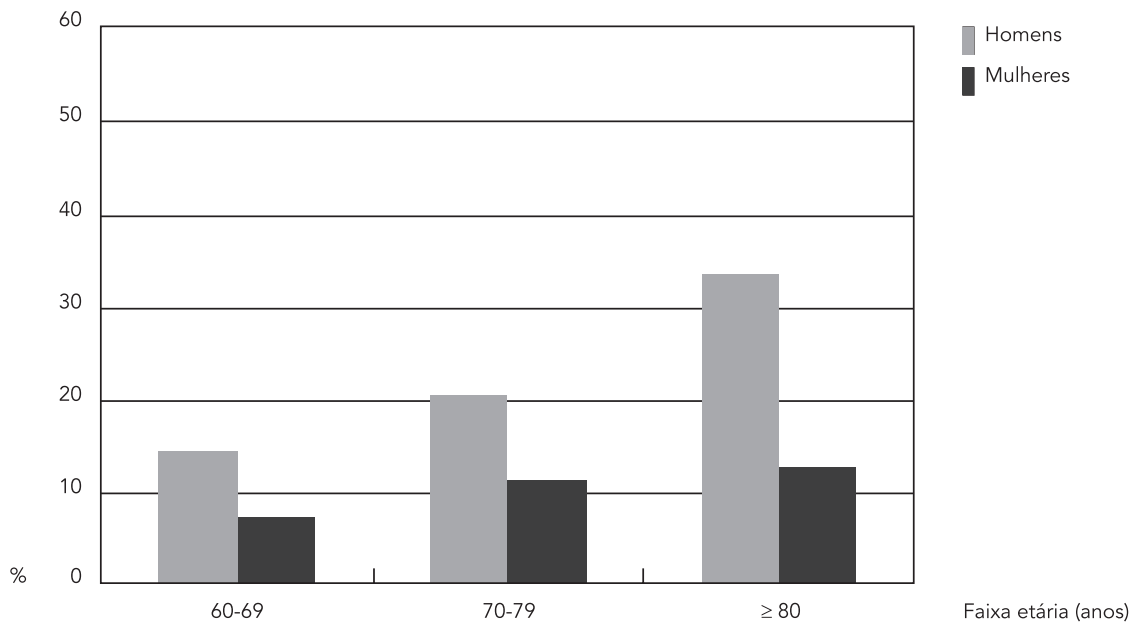

em relação às características sociodemográficas, condições de saúde e uso de serviços de saúde, tomando-se os eutróficos como categoria de referência. O baixo peso se associou de forma positiva e significante com a renda menor ou igual à mediana $(\mathrm{p}<0,001)$, com a pior percepção de saúde $(\mathrm{p}=0,021)$, e negativamente com história de dislipidemias $(0,032)$. O excesso de peso foi associado positiva e significantemente com sexo feminino ( $\mathrm{p}<0,001)$, história de diabetes $(\mathrm{p}=$ $0,009)$, história de hipertensão ( $\mathrm{p}=0,019)$, história de dislipidemia $(\mathrm{p}=0,004)$, história de artrite/ artrose $(p=0,003)$, número de doenças autorrelatadas ( $\mathrm{p}<0,001$ ), polifarmácia (uso de 5 ou mais 


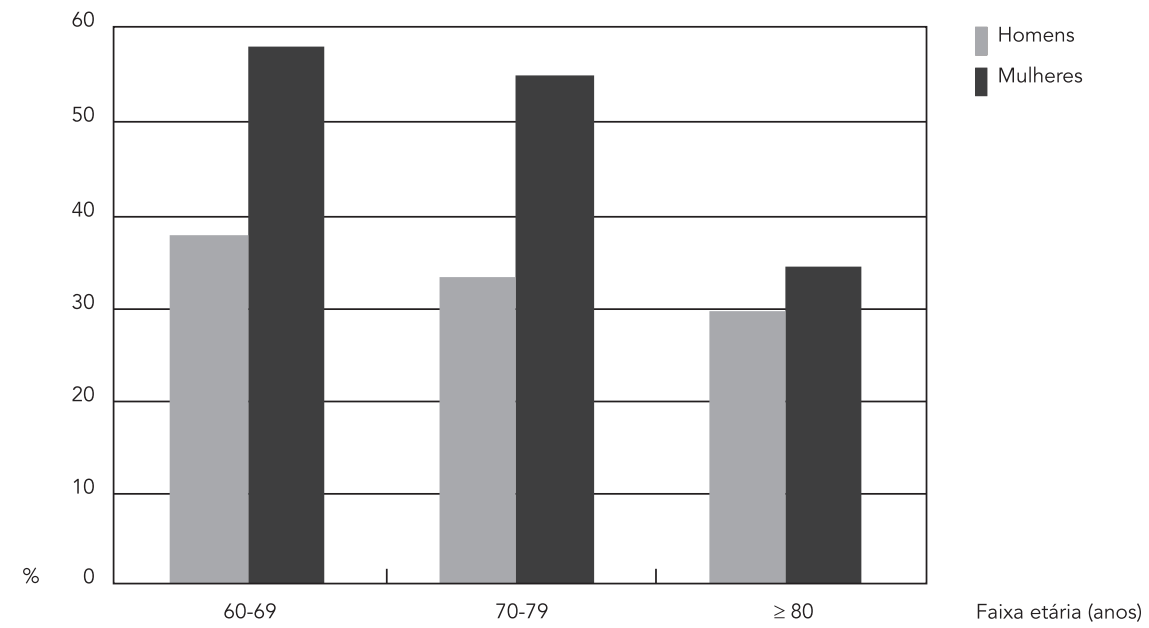

medicamentos) ( $\mathrm{p}=0,010)$, e negativamente ao tabagismo $(\mathrm{p}=0,001)$.

Os resultados da análise multivariada (Tabela 3) da associação entre baixo peso e excesso de peso em relação às características sociodemográficas, condições de saúde e uso de serviços de saúde mostram que a pior percepção da saúde se manteve independentemente associada ao baixo peso. Os fatores independentemente associados ao excesso de peso foram sexo feminino, idade igual ou superior a 80 anos, tabagismo e história de artrite/artrose.

\section{Discussão}

No presente estudo, o sexo feminino apresentou maior valor de IMC em comparação ao masculino $(p<0,001)$. Esses resultados estão em concordância com a literatura nacional 7,13,14 e de outros países como Itália, Espanha, Chile, México, Cuba e Coreia do Sul 4,15,16,17,18,19. Com o aumento da idade observou-se redução significante do IMC somente entre os homens. Em outros estudos, esse fenômeno ocorreu em ambos os sexos 4,15,16.

Nossos resultados evidenciam considerável prevalência de excesso de peso entre os idosos estudados. Esses achados são consistentes com os observados entre os idosos de Pelotas, Rio Grande do Sul 20. Com relação ao sexo, diferentes estudos mostram que o excesso de peso é mais prevalente entre as idosas, em contraste com o maior número de homens idosos com baixo peso 5,6,20,21. Barreto et al. 7, em estudo realizado em Bambuí, Minas Gerais, com 1.742 idosos, observaram redução da prevalência de excesso de peso dentre as idosas em faixa etária mais elevada, conforme resultados apresentados neste estudo. Essa menor prevalência de excesso de peso pode estar associada às mudanças da composição corporal que varia entre os sexos nesse grupo etário. Dentre as mulheres, o maior ganho de peso atinge um platô aos 75 anos e geralmente a partir daí começa a declinar. Já com os homens esse platô ocorre em torno dos 65 anos 11.

No modelo final da análise multivariada, o sexo feminino manteve-se associado de forma independente ao excesso de peso conforme evidenciaram outros estudos 7,8. O excesso de peso entre as idosas pode estar relacionado à maior expectativa de vida e ao maior acúmulo de gordura visceral ${ }^{12}$. As modificações em termos de redistribuição da gordura corporal levam ao maior acúmulo de gordura visceral e intra-abdominal, enquanto a gordura subcutânea tende a diminuir nos membros 2,3. Esse acúmulo de gordura visceral ocorre em idades avançadas mais nas mulheres do que nos homens, além disso, as mulheres acumulam mais gordura total. Outra explicação referida na literatura é a menopausa, que é acompanhada por aumento de peso e adiposidade 11 .

Outro fator independente e negativamente associado ao excesso de peso foi a idade maior 
Análise bivariada da associação entre as condições sociodemográficas e de saúde e o estado nutricional dos idosos. Viçosa, Minas Gerais, Brasil, 2009 *.

\begin{tabular}{|c|c|c|c|c|c|c|}
\hline \multirow[t]{2}{*}{ Variáveis } & \multicolumn{2}{|c|}{ Baixo peso } & \multirow[t]{2}{*}{ Valor de $p$} & \multicolumn{2}{|c|}{ Excesso de peso } & \multirow[t]{2}{*}{ Valor de $p$} \\
\hline & n (\%) & OR (IC95\%) & & n (\%) & OR (IC95\%) & \\
\hline \multicolumn{7}{|l|}{ Sexo } \\
\hline Masculino & $50(18,2)$ & 1,0 & & $97(35,4)$ & 1,0 & \\
\hline Feminino & $25(9,0)$ & $0,6(0,4-1,1)$ & 0,096 & $151(54,5)$ & $1,9(1,3-2,8)$ & $<0,001$ \\
\hline \multicolumn{7}{|l|}{ Faixa etária (anos) } \\
\hline $60-69$ & $32(10,5)$ & 1,0 & & $147(48,4)$ & 1,0 & \\
\hline $70-79$ & $30(16,0)$ & $1,5(0,9-2,7)$ & 0,139 & $82(43,6)$ & $0,9(0,6-1,3)$ & 0,667 \\
\hline 80 e mais & $13(22,0)$ & $1,8(0,9-4,0)$ & 0,107 & $19(32,2)$ & $0,6(0,3-1,1)$ & 0,112 \\
\hline \multicolumn{7}{|l|}{ Escolaridade } \\
\hline Nunca estudou & $11(13,9)$ & 1,0 & & $40(50,6)$ & 1,0 & \\
\hline Até séries iniciais do Ensino Fundamental & $54(15,4)$ & $0,9(0,4-2,0)$ & 0,863 & $150(42,7)$ & $0,7(0,4-1,2)$ & 0,217 \\
\hline Séries finais do Ensino Fundamental ou mais & $10(8,3)$ & $0,5(0,2-1,3)$ & 0,150 & $58(48,3)$ & $0,8(0,4-1,4)$ & 0,427 \\
\hline \multicolumn{7}{|l|}{ Renda mensal individual ** (mediana) } \\
\hline Maior que a mediana & $23(8,78)$ & 1,0 & & $118(45,03)$ & 1,0 & \\
\hline Menor ou igual à mediana & $45(38,43)$ & $2,5(1,4-4,5)$ & 0,001 & $104(42,98)$ & $1,1(0,7-1,6)$ & 0,477 \\
\hline \multicolumn{7}{|l|}{ Tabagismo } \\
\hline Nunca fumou & $37(12,6)$ & 1,0 & & $146(49,7)$ & 1,0 & \\
\hline Ex-fumante & $22(11,4)$ & $0,8(0,4-1,4)$ & 0,478 & $89(46,1)$ & $0,8(0,5-1,2)$ & 0,332 \\
\hline Fuma atualmente & $16(25,0)$ & $1,4(0,7-2,7)$ & 0,376 & $13(20,3)$ & $0,3(0,1-0,5)$ & $<0,001$ \\
\hline \multicolumn{7}{|l|}{ Atividade física } \\
\hline Nã & $67(15,2)$ & 1,0 & & $165(44,1)$ & 1,0 & \\
\hline Sim & $18(10,2)$ & $0,6(0,3-1,1)$ & 0,975 & $83(46,9)$ & $1,0(0,7-1,5)$ & 0,132 \\
\hline \multicolumn{7}{|l|}{ Autopercepção da saúde } \\
\hline Muito boa/Boa & $28(11,4)$ & 1,0 & & $112(45,5)$ & 1,0 & \\
\hline Regular & $37(14,0)$ & $1,2(0,7-2,2)$ & 0,432 & $115(43,6)$ & $0,9(0,7-1,4)$ & 0,880 \\
\hline Ruim/Muito ruim & $7(23,3)$ & $3,8(1,2-11,7)$ & 0,021 & $16(53,3)$ & $2,2(0,8-5,5)$ & 0,103 \\
\hline \multicolumn{7}{|l|}{ História de diabetes } \\
\hline Não & $83(14,8)$ & 1,0 & & $177(41,5)$ & 1,0 & \\
\hline Sim & $12(9,6)$ & $0,8(0,4-1,7)$ & 0,635 & $71(56,8)$ & $1,7(1,1-2,7)$ & 0,009 \\
\hline \multicolumn{7}{|l|}{ História de hipertensão } \\
\hline Não & $26(19,3)$ & 1,0 & & $46(34,1)$ & 1,0 & \\
\hline $\operatorname{Sim}$ & $49(11,8)$ & $0,7(0,4-1,2)$ & 0,247 & $202(48,6)$ & $1,7(1,1-2,6)$ & 0,019 \\
\hline \multicolumn{7}{|l|}{ História de dislipidemias } \\
\hline Não & $46(19,4)$ & 1,0 & & $84(35,4)$ & 1,0 & \\
\hline Sim & $29(9,2)$ & $0,5(0,3-0,9)$ & 0,032 & $164(52,2)$ & $1,7(1,2-2,5)$ & 0,004 \\
\hline \multicolumn{7}{|l|}{ História de artrite/artrose } \\
\hline Não & $65(15,6)$ & 1,0 & & $169(40,5)$ & 1,0 & \\
\hline Sim & $10(7,5)$ & $0,6(0,3-1,3)$ & 0,215 & $79(59,0)$ & $1,9(1,2-2,9)$ & 0,003 \\
\hline \multicolumn{7}{|l|}{ Número de doenças } \\
\hline Até 4 & $54(15,2)$ & 1,0 & & $139(39,2)$ & 1,0 & \\
\hline 5 ou mais & $21(10,7)$ & $0,9(0,8-1,0)$ & 0,130 & $109(55,6)$ & $1,2(1,1-1,3)$ & $<0,001$ \\
\hline \multicolumn{7}{|l|}{ Número de medicamentos usados nos últimos } \\
\hline \multicolumn{7}{|l|}{15 dias } \\
\hline Até 4 & $58(15,8)$ & 1,0 & & $148(44,0)$ & 1,0 & \\
\hline 5 ou mais & $17(9,3)$ & $0,7(0,4-1,3)$ & 0,291 & $100(36,1)$ & $1,6(1,1-2,4)$ & 0,010 \\
\hline \multicolumn{7}{|l|}{ Número de hospitalizações nos últimos 12 meses } \\
\hline 0 & $65(13,8)$ & 1,0 & & $205(43,6)$ & 1,0 & \\
\hline 1 ou mais & $10(12,5)$ & $1,1(0,5-2,4)$ & 0,811 & $42(52,5)$ & $1,4(0,9-2,4)$ & 0,148 \\
\hline
\end{tabular}

IC95\%: intervalo de 95\% de confiança; OR: odds ratio.

* Participaram desta análise 551 idosos. Para esta análise, a categoria de referência foi a eutrofia;

** Valor mediano da renda à época do estudo: $\mathrm{R} \$ 697,75$. 
Resultados finais da análise multivariada da associação entre as condições sociodemográficas e de saúde com o estado nutricional dos idosos. Viçosa, Minas Gerais, Brasil, 2009 *.

\begin{tabular}{|c|c|c|}
\hline \multirow[t]{2}{*}{ Variáveis } & Baixo peso & Excesso de peso \\
\hline & OR (IC95\%) ** & OR (IC95\%) ** \\
\hline \multicolumn{3}{|l|}{ Sexo } \\
\hline Masculino & 1,0 & 1,0 \\
\hline Feminino & $0,7(0,4-1,2)$ & $1,5(1,0-2,3)$ \\
\hline \multicolumn{3}{|l|}{ Faixa etária (anos) } \\
\hline $60-69$ & 1,0 & 1,0 \\
\hline $70-79$ & $1,6(0,9-3,05)$ & $0,8(0,6-1,3)$ \\
\hline 80 e mais & $1,6(0,7-3,4)$ & $0,3(0,1-0,6)$ \\
\hline \multicolumn{3}{|l|}{ Tabagismo } \\
\hline Nunca fumou & 1,0 & 1,0 \\
\hline Ex-fumante & $0,9(0,4-2,3)$ & $1,2(0,7-2,2)$ \\
\hline Fuma atualmente & $0,5(0,7-2,9)$ & $0,3(0,1-0,6)$ \\
\hline \multicolumn{3}{|c|}{ Autopercepção da saúde } \\
\hline Muito boa/Boa & 1,0 & 1,0 \\
\hline Regular & $1,4(0,8-2,7)$ & $0,8(0,5-1,2)$ \\
\hline Ruim/Muito ruim & $3,3(1,1-10,0)$ & $2,0(0,8-5,3)$ \\
\hline \multicolumn{3}{|c|}{ História de artrite/Artrose } \\
\hline Não & 1,0 & 1,0 \\
\hline Sim & $0,6(0,3-1,4)$ & $1,7(1,1-2,7)$ \\
\hline
\end{tabular}

IC95\%: intervalo de 95\% de confiança; OR: odds ratio.

* Participaram desta análise 551 idosos;

** OR e IC95\% ajustados pelo método de regressão logística multinomial por todas as variáveis apresentadas na tabela. Para esta análise, a categoria de referência foi a eutrofia.

ou igual a 80 anos. Estudos realizados no Brasil evidenciaram essa associação 6,7. A redução do excesso de peso com o envelhecimento pode ser explicada, em parte, pelo efeito do viés de sobrevivência, devido à obesidade e às patologias a ela associadas contribuírem para mortalidade dos idosos obesos antes dos 80 anos 3,14. Outra possível explicação se deve ao efeito de coorte, pois parte-se do princípio de que a obesidade contribui para o aparecimento de doenças, e que essas levam à perda de peso involuntária. Esse fato pode levar à inclusão de idosos eutróficos na coorte, que já tiveram sobrepeso ou obesidade na meia idade. Esse viés resultaria em um aumento do risco de mortalidade no grupo dos eutróficos e diminuiria o risco relativo de mortalidade no grupo dos obesos 22 .

Outra variável independentemente associada de forma positiva com o excesso de peso foi a artrite/artrose, e esse achado está de acordo com outros estudos 9,23. É provável que o mecanismo pelo qual o excesso de peso aumenta o risco de osteoartrite esteja relacionado à sobrecarga mecânica 24,25 , e a diminuição do risco de pro- gressão da doença esteja associada à redução do peso 25 . $\mathrm{O}$ aumento de carga durante a atividade leva ao aumento do estresse sobre a cartilagem e induz a degradação dos tecidos levando à osteoartrite 25 . Além do efeito mecânico, outro fator que pode explicar o elo entre osteoartrite e excesso de peso é o hormônio leptina. Níveis plasmáticos de leptina estão fortemente correlacionados com a massa gorda e esses níveis diminuem com a perda de peso. Verifica-se, nos adultos, receptores de leptina nos condrócitos articulares e esta também pode desempenhar um papel no desenvolvimento da osteoartrite através da matriz óssea 23 .

A pior percepção do estado de saúde manteve-se associada ao baixo peso no modelo final. Resultado semelhante foi encontrado no estudo realizado em 2003 por Barreto et al. 7 em Bambuí. Com relação ao excesso de peso (IMC $\geq 30 \mathrm{~kg}$ / $\mathrm{m}^{2}$ ), esses autores também observaram que este esteve associado à pior percepção de saúde, diferentemente do resultado encontrado neste trabalho. Esse fato pode ser devido aos pontos de cortes adotados pelos estudos, os quais foram di- 
ferentes. Não existe um consenso sobre o ponto de corte de IMC adequado para os idosos. Esses diferentes pontos de corte dificultam a interpretação e a comparação com outros trabalhos.

Neste estudo, o tabagismo se mostrou como um fator independente e negativamente associado ao excesso de peso. Resultado semelhante foi encontrado por Kaplan et al. 26 em pesquisa realizada no Canadá com 8.802 idosos, utilizando os pontos de corte da Organização Mundial da Saúde (OMS), na qual observaram que o tabagismo foi associado negativamente com o sobrepeso (OR = 0,62; IC95\%: 0,56-0,70) e a obesidade $(\mathrm{OR}=0,56$; IC95\%: 0,51-0,61). Normalmente, os fumantes são mais magros e têm mais elevada mortalidade quando comparados aos não fumantes, sendo que o aumento da mortalidade está associado ao menor peso em função do tabagismo 22,25. Esse efeito pode ser mais pronunciado nos idosos, pois eles fumaram por um longo período da vida quando comparados aos jovens e adultos 22. Nos Estados Unidos, Adams et al. 27 encontraram associação entre a obesidade e maior risco de morte nos idosos que nunca fumaram do que entre os ex-fumantes e fumantes. Barreto et al. 7 não encontraram associação entre fumar e ser ex-fumante com a obesidade em Bambuí. Janssen \& Mark 28, em revisão sistemática observaram que o efeito do tabagismo pode aumentar a mortalidade entre os idosos com menor IMC, sendo esse risco menor entre aqueles com maiores valores de IMC. Estudos longitudinais são necessários para avaliar o efeito do tempo sobre os altos valores do IMC em relação à história de tabagismo.

\section{Considerações finais}

Os resultados deste estudo evidenciam um cenário caracterizado pela prevalência elevada de excesso de peso, sobretudo entre as mulheres e aqueles que relataram artrite/artrose. Uma limitação do desenho transversal é a impossibilidade de se definir ao certo se o excesso de peso leva à doença ou se a doença leva ao excesso de peso entre os idosos. Dessa forma, a prevenção de doenças e o controle do peso com o avanço da idade são ações em saúde de grande importância para manutenção de uma vida saudável. Contrariamente, o baixo peso parece ser mais importante entre os homens, sobretudo naqueles mais idosos, e esteve associado à pior percepção da saúde. Os idosos são mais vulneráveis ao baixo peso devido ao processo de envelhecimento que está relacionado a mudanças da composição corporal, ficando assim mais propensos aos riscos decorrentes da perda de peso. Diante desses resultados, esforços devem ser feitos para aprimorar as políticas públicas direcionadas para esse grupo etário por meio da intervenção no baixo peso e excesso de peso, bem como na melhoria dos seus hábitos de vida. Vale ainda ressaltar estratégias como atividades sociais e culturais para favorecerem mudanças no estilo de vida, tais como a adoção da prática de atividade física e de hábitos alimentares saudáveis. Nesse sentido, a identificação e consideração dos fatores associados adquirem grande relevância no âmbito da Saúde Coletiva.

\footnotetext{
Resumo

Investigaram-se as prevalências de baixo peso e excesso de peso, bem como os fatores associados em idosos. Trata-se de um estudo transversal, com 621 idosos residentes no Município de Viçosa, Minas Gerais, Brasil, realizado em 2009. As informações foram obtidas por meio de entrevistas domiciliares e se referiam à avaliação do estado nutricional, condições sociodemográficas e de saúde. Do total de pessoas estudadas, 53,3\% eram do gênero feminino. A mediana do IMC foi de $26,40 \mathrm{~kg} /$ $m^{2}$ (mínima 15,20 e máxima 46,82) e foi menor para os homens do que para as mulheres. A prevalência de excesso de peso foi elevada (45\%; IC95\%: 40\%-49\%),
}

diminuiu com a idade, associou-se positivamente ao gênero feminino e história de artritelartrose, e negativamente à faixa etária de 80 anos ou mais e ao tabagismo. A prevalência de baixo peso foi maior no gênero masculino (18,2\%), aumentou com o avanço da idade e associou-se positivamente à pior percepção de saúde. O conjunto dos resultados salientam a importância de estratégias em saúde que favoreçam mudanças no estilo de vida e hábitos alimentares saudáveis.

Estado Nutricional; Índice de Massa Corporal; Obesidade; Idoso 


\section{Colaboradores}

C. M. Nascimento e S. C. C. Franceschini contribuíram na concepção, projeto, análise e interpretação dos dados; redação do artigo e revisão crítica relevante do conteúdo intelectual; e aprovação final da versão a ser publicada. A. Q. Ribeiro colaborou no projeto, análise e interpretação dos dados; redação do artigo e revisão crítica relevante do conteúdo intelectual; e aprovação final da versão a ser publicada. R. M. M. Cotta e S. E. Priore participaram da redação do artigo e revisão crítica relevante do conteúdo intelectual. F. A. Acurcio e S. V. Peixoto colaboraram na revisão crítica relevante do conteúdo intelectual.

\section{Agradecimentos}

Ao Conselho Nacional de Desenvolvimento Científico e Tecnológico (processos: 474689-2008-5 e 579255/2008-5) pela concessão da bolsa de mestrado e financiamento deste projeto. À Coordenação de Aperfeiçoamento de Pessoal de Nível Superior (processo $\mathrm{n}^{\circ}$. 23038.039412/2008-73) pela concessão da bolsa de pós-doutorado e financiamento deste projeto.

\section{Referências}

1. Lima-Costa MF, Veras R. Saúde pública e envelhecimento [Editorial]. Cad Saúde Pública 2003; 19:700-1.

2. Lebrão ML. O envelhecimento no Brasil: aspectos da transição demográfica e epidemiológica. Saúde Coletiva 2007; 4:135-40.

3. Seidell, JC, Visscher TLS. Body weight and weight change and their health implications for the elderly. Eur J Clin Nutr 2000; 54:33-9.

4. Perissinotto E, Pisent C, Sergi G, Grigoletto F, Enzi G. Anthropometric measurements in the elderly: Age and gender differences. Br J Nutr 2002; 87:17786.

5. Tavares EL, Anjos LA. Perfil antropométrico da população idosa brasileira. Resultados da Pesquisa Nacional sobre Saúde e Nutrição. Cad Saúde Pública 1999; 15:759-68.

6. Campos MAG, Pedroso ERP, Lamounier JA, Colosimo EA, Abrantes MM. Estado nutricional e fatores associados em idosos. Rev Assoc Med Bras (1992) 2006; 52:214-21.

7. Barreto SM, Passos VMA, Lima-Costa MFF. Obesity and underweight among Brazilian elderly. The Bambuí health and aging study. Cad Saúde Pública 2003; 19:605-12.
8. Da Cruz IBM, Almeida MSC, Schwanke CHA, Moriguchi EH. Prevalência de obesidade em idosos longevos e sua associação com fatores de risco e morbidades cardiovasculares. Rev Assoc Med Bras (1992) 2004; 50:172-7.

9. Machado GPM, Barreto SM, Passos VMA, LimaCosta MF. Projeto Bambuí: prevalência de sintomas articulares crônicos em idosos. Rev Assoc Med Bras (1992) 2004; 50:367-72.

10. World Health Organization. Physical status: the use and interpretation of anthropometry. Geneva: World Health Organization; 1995. (Technical Report Series, 854).

11. Lipschitz DA. Screening for nutritional status in the elderly. Prim Care 1994; 21:55-67.

12. Pagano M, Gauvreau K. Princípios de bioestatística. 2a Ed. São Paulo: Cengage Learning; 2008.

13. Cabrera MAS, Jacob-Filho W. Obesidade em idosos: prevalência, distribuição e associação com hábitos e co-morbidades. Arq Bras Endocrinol Metab 2001; 45:494-501.

14. Barbosa AR, Souza JMP, Lebrão ML, Laurenti R, Marucci MFN. Anthropometry of elderly residents in the city of São Paulo, Brazil. Cad Saúde Pública 2005; 21:1929-38. 
15. Gutiérrez-Fisac JL, López E, Banegas JR, Graciani A, Rodríguez-Artalejo F. Prevalence of overweight and obesity in elderly people in Spain. Obes Res 2004; 12:710-5.

16. Santos JL, Albala C, Lera L, Garcia C, Arroyo P, Pérez-Bravo F, et al. Anthropometric measurements in the elderly population of Santiago, Chile. Nutrition 2004; 20:452-7.

17. Sánchez-Garcia S, García-Peña C, Duque-López MX, Juárez-Cedillo T, Cortés-Núñez AR, ReyesBeaman S. Anthropometric measures and nutritional status in a healthy elderly population. BMC Public Health 2007; 7:1-9.

18. Coqueiro RS, Barbosa AR, Borgatto AF. Anthropometric measurements in the elderly of Havana, Cuba: Age and sex differences. Nutrition 2009; 25:33-9.

19. Han SS, Kim KW, Kim K, Na KY, Chae DW, Kim S, et al. Lean mass index: a better predictor of mortality than body mass index in elderly Asians. J Am Geriatr Soc 2009; 8:312-7.

20. Silveira EA, Kac G, Barbosa LS. Prevalência e fatores associados à obesidade em idosos residentes em Pelotas, Rio Grande do Sul, Brasil: classificação da obesidade segundo dois pontos de corte do índice de massa corporal. Cad Saúde Pública 2009; 25:1569-77.

21. Silva CLA. Fatores associados ao estado nutricional e ao nível de hemoglobina em idosos: Programa de Saúde da Família, Viçosa - MG [Dissertação de Mestrado]. Viçosa: Universidade Federal de Viçosa; 2008.
22. Janssen I, Bacon E. Effect of current and midlife obesity status on mortality risk in the elderly. Obesity (Silver Spring) 2008; 16:2504-9.

23. Reijamn M, Pols HAP, Bergink AP, Hazes JMW, Belo JN, Lievense AM, et al. Body mass index associated with onset and progression of osteoarthritis of the knee but not of the hip: The Rotterdam Study. Ann Rheum Dis 2007; 66:158-62.

24. Janssen I, Bacon E. Effect of current and midlife obesity status on mortality risk in the elderly. Obesity (Silver Spring) 2007; 5:763-78.

25. Zamboni M, Mazzali G, Zoico E, Harris TB, Meigs JB, Di Francesco V, et al. Health consequences of obesity in the elderly: a review of four unresolved questions. Int J Obes 2005; 29:1011-29.

26. Kaplan MS, Huguet N, Newson JT, McFarland BH, Lindsay J. Prevalence and correlates of overweight and obesity among older adults: findings from the Canadian national population health survey. J Gerontol A Biol Sci Med Sci 2003; 58:1018-30.

27. Adams KF, Schatzkin A, Harris T, Kipnis V, Mouw T, Barbash RB, et al. Overweight, obesity, and mortality in a large prospective cohort of persons 50 to 71 years old. N Engl J Med 2006; 355:763-78.

28. Janssen I, Mark AE. Elevated body mass index and mortality risk in the elderly. Obes Rev 2007; 8:41-59.

Recebido em 21/Dez/2010

Versão final reapresentada em 20/Jul/2011

Aprovado em 15/Set/2011 